\title{
A IMPORTÂNCIA DO ANEL DE SILICONE NA DERIVAÇÃO GÁSTRICA EM Y-DE-ROUX PARA O TRATAMENTO DA OBESIDADE
}

\author{
THE IMPORTANCE OF THE SILASTIC RING IN THE ROUX-EN-Y GASTRIC BYPASS FOR \\ THE TREATMENT OF OBESITY
}

\author{
Antonio Carlos Valezi, TCBC-PR ${ }^{1}$; Edivaldo Macedo de Brito,ACBC-PR²; \\ José Carlos Lacerda de Souza, ACBC-PR²; Ana Luiza Mezzaroba Guariente ${ }^{3}$; \\ Fabiano Takaaki Emori³; Vivian Cristina Holanda Lopes
}

\begin{abstract}
RESUMO: Objetivo: Analisaram prospectivamente pacientes obesos submetidos à derivação gástrica em y-de-roux com anel de silicone de diferentes tamanhos. Método: Os pacientes foram acompanhados durante cinco anos e através de exame endoscópico foram classificados em dois Grupos: anel com diâmetro interno até $10 \mathrm{~mm}$ (184 pacientes) e anel com diâmetro interno maior que $10 \mathrm{~mm}$ (107pacientes). Foi mensurada a porcentagem de perda do excesso de peso com um, dois e cinco anos de pós-operatório, e comparados os grupos entre si em cada ano da coleta dos dados. Resultados: Houve maior emagrecimento no grupo com anel de diâmetro interno até $10 \mathrm{~mm}$. A análise estatística mostrou que houve significância comparando-se os dois grupos entre si, um, dois e cinco anos após a cirurgia, sendo o emagrecimento sempre maior no grupo com anel de menor diâmetro. Não houve diferença estatisticamente significante ao se comparar outros dados da amostra como idade, sexo e IMC. Conclusão: A restrição determinada pelo anel aumenta a porcentagem de perda do excesso de peso (Rev. Col. Bras. Cir. 2008; 35(1): 018-022).
\end{abstract}

Descritores: Gastroplastia; Obesidade mórbida; Cirurgia bariátrica.

\section{INTRODUÇÃO}

Estima-se que $98 \%$ dos pacientes com obesidade classe III que conseguem emagrecer retornam ao peso pré-tratamento em menos de um ano, e $100 \%$ o fazem em cinco anos, independente do tratamento conservador escolhido ${ }^{1}$. Atualmente o tratamento cirúrgico é o único que acarreta uma substancial e duradoura perda de peso e também uma melhora das comorbidades $^{2,3}$.

Mason \& $\mathrm{Ito}^{4}$, em 1967, introduziram o conceito da redução gástrica no tratamento da obesidade. O estômago era seccionado horizontalmente próximo ao fundo gástrico, criando-se reservatório de $100 \mathrm{ml}$ de capacidade e era feita gastrojejunostomia de $20 \mathrm{~mm}$ na grande curvatura. Mason \& Printen 5 , em 1972, modificaram esse procedimento propondo anastomose mais estreita, entre 8 e $12 \mathrm{~mm}$, por entenderem que isto aumentaria o emagrecimento. Iniciava-se aí a controvérsia sobre a necessidade ou não de algum método de restrição ao esvaziamento gástrico para a obtenção de melhores resultados cirúrgicos. Em 1977, Griffen ${ }^{6}$ et al., realizaram gastrojejunostomia em Y-de-Roux , e não mais em alça, que prevenia o refluxo biliar para a bolsa gástrica. Torres, Oca e Garrison ${ }^{7}$ passaram a confeccionar bolsas gástricas verticais, junto à pequena curvatura, local em que as bolsas se dilatam menos, associadas à gastroenterostomia de $1,8 \mathrm{~cm}$ de diâmetro.

O uso de próteses na porção terminal da bolsa gástrica teve início com Laws \& Piantadosi ${ }^{8}$, em 1981, e com Linner \& Drew ${ }^{9}$, em 1985, que empregaram anel de silicone como elemento de contenção para prevenir a dilatação da gastroenterostomia que acontecia com o tempo. Nascia aí outro ponto de debate no tratamento cirúrgico da obesidade, que é a necessidade de material protético para o retardo do esvaziamento gástrico, uma vez que a anastomose dilata-se com o tempo, mesmo se realizada com grampeadores. Fobi ${ }^{10}$, em 1986, idealizou a cirurgia que consistia na realização de bolsa gástrica junto à pequena curvatura gástrica associada à jejunojejunostomia a $100 \mathrm{~cm}$ da gastroenterostomia que era realizada de maneira ampla, uma vez que acima dessa anastomose era colocado anel de silicone. Capella ${ }^{11}$, em 1990, descreveu procedimento semelhante, colocando também anel de silicone no extremo distal da bolsa. Posteriormente, substituiu o anel por fita de polipropileno, advertindo, entretanto, que era necessário um fator de contenção ao esvaziamento gástrico rápido para obtenção de bons resultados em relação à perda de peso. As modificações da derivação gástrica, propostas por Fobi ${ }^{10}$ e Capella, ${ }^{11}$, tornaram-se referências no tratamento cirúrgico da obesidade.

1. Professor Associado Doutor do Departamento de Cirurgia da Universidade Estadual de Londrina - PR

2. Professor Adjunto Mestre do Departamento de Cirurgia da Universidade Estadual de Londrina - PR.

3. Acadêmicos de Medicina da Universidade Estadual de Londrina - PR.

Recebido em 16/08/2007

Aceito para publicação em 19/10/2007

Conflito de interesses: nenhum

Fonte de financiamento: nenhuma

Trabalho realizado no Hospital Universitário da Universidade Estadual de Londrina - PR. 
Devido às controvérsias que existem sobre este assunto, resolvemos medir o diâmetro interno do anel de silicone, na derivação gástrica em Y-de-Roux, e analisar se o diâmetro tem relação com a perda de peso pós-operatória do paciente.

\section{MÉTODO}

O estudo foi realizado no Hospital Universitário da Universidade Estadual de Londrina. Analisaram-se pacientes operados entre maio de 1999 e dezembro de 2000. A pesquisa teve início após aprovação pela Comissão de Ética do hospital.

Foram operados 327 pacientes obesos, considerados aptos após avaliação clínica por equipe multidisciplinar que inclui endocrinologista, cardiologista, pneumologista, nutricionista, psicólogo, psiquiatra e assistente social.

Todos os pacientes incluídos no estudo, foram submetidos à derivação gástrica em Y-de-Roux, que consiste em gastroplastia vertical junto à pequena curvatura, com $10 \mathrm{~cm}$ de comprimento e $4,5 \mathrm{~cm}$ de largura; colocação de anel de silicone no terço distal da bolsa gástrica com 6,2 ou $6,5 \mathrm{~cm}$ de comprimento; gastroenterostomia manual ampla e jejunojejunostomia término-lateral distante $100 \mathrm{~cm}$ da gastroenterostomia. $\mathrm{O}$ anel de silicone era ajustado sobre sonda de $10 \mathrm{~mm}$ de diâmetro, colocado no interior da bolsa gástrica. Todos os procedimentos foram realizados pela mesma equipe, cujo treinamento inicial foi idêntico. As operações foram feitas pela via laparotômica.

Os pacientes foram acompanhados ambulatorialmente. Em cada retorno eram avaliados pela equipe multidisciplinar, liderados sempre pelo mesmo cirurgião. O acompanhamento completo foi conseguido em 291 pacientes.

Os paciente foram distribuidos em dois grupos após o exame endoscópico: - anel de diâmetro interno até $10 \mathrm{~mm}$, 184 pacientes; - anel de diâmetro interno maior que 10 mm, 107 pacientes.

Os dados deste estudo foram coletados prospectivamente pelos profissionais envolvidos no atendimento e analisados retrospectivamente. Os dados referem-se àqueles do pré-operatório, um ano, dois e cinco anos de pós-operatório para a perda de peso.

Analisamos a idade, o índice de massa corporal (IMC), a perda de peso e a falha do tratamento cirúrgico. A perda de peso foi avaliada pela perda do excesso de peso medida em porcentagem. $\mathrm{Na}$ análise da perda do excesso de peso estratificamos a amostra em Grupo composto por pacientes com anel de diâmetro interno até 10mm (184 pacientes) e em outro composto por pacientes com anel de diâmetro interno maior que $10 \mathrm{~mm}$ (107 pacientes). Comparamos a perda de peso de cada grupo, um, dois e cinco anos após a operação.

Analisamos a falha do tratamento cirúrgico em função da perda de peso. Foi considerada como falha aquele paciente que perdeu menos do que $50 \%$ do excesso de peso em 5 anos de acompanhamento, conforme critérios adotados por MacLean ${ }^{12}$, Capella $^{13}$ e Fobi ${ }^{14}$.

Os pacientes foram submetidos a exame endoscópico rotineiramente um ano após a cirurgia. $\mathrm{O}$ exame foi sempre realizado pelo mesmo endoscopista, que possui conhecimen- to técnico sobre a operação. Após o exame, os pacientes foram distribuidos em dois grupos, aqueles com diâmetro interno do anel até $10 \mathrm{~mm}$ e aqueles com diâmetro além de $10 \mathrm{~mm}$. O endoscopista fazia a medida, utilizando endoscópio com diâmetro de $10 \mathrm{~mm}$, assim se o aparelho passasse sem esforço pela constrição determinada pelo anel, o paciente era colocado num grupo, caso houvesse alguma resistência à progressão do aparelho o paciente era enquadrado em outro grupo.

Os pacientes dos dois grupos foram comparados entre si, com a análise dos seguintes parâmetros:

- idade - IMC pré e pós-operatório - perda do excesso de peso, um, dois e cinco anos após a operação.

Cada Grupo foi comparado com seu correspondente, analisando-se os parâmetros já citados acima, a fim de estabelecermos a relação entre o diâmetro interno do anel e a evolução pós-operatória para os parâmetros analisados.

Foi utilizado o teste t pareado comparando os grupos entre si, e considerando p estatisticamente significante se menor que 0,0001 .

\section{RESULTADOS}

Comparando-se a prevalência de homens e mulheres no grupo com anel menor e maior que $10 \mathrm{~mm}$, não houve diferença estatística. Não se observou diferença estatística ao se comparar a idade dos grupos com anel maior e menor que $10 \mathrm{~mm}$. Não houve diferença significante do ponto de vista estatístico ao se comparar os grupos em relação ao IMC.

Ao se analisar os resultados da perda do excesso do peso após um ano da operação, houve diferença estatisticamente significante entre os dois grupos, sendo maior no Grupo com anel até $10 \mathrm{~mm}$ de diâmetro. A comparação da perda do excesso de peso, entre os grupos, dois anos após a operação mostra que esta foi maior no grupo com anel até $10 \mathrm{~mm}$, com diferença estatisticamente significante.

$\mathrm{Na}$ análise da perda do excesso de peso cinco anos após a operação também houve diferença com significância estatística, sendo a perda do excesso de peso maior no grupo com anel de diâmetro inferior.

A Figura 1 ilustra a diferença de perda do excesso de peso entre os dois grupos, sendo sempre maior no grupo com anel menor que $10 \mathrm{~mm}$, e também sempre com diferença estatis-

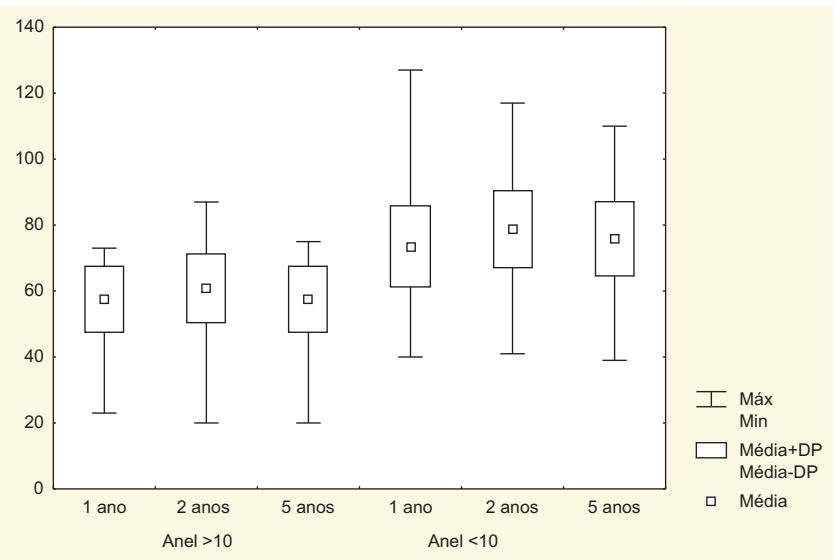

Figura 1 - Perda do excesso de peso após um, dois e cinco anos da cirurgia. 
ticamente significante ao longo das análises de um, dois e cinco anos após a operação.

Houve falha do tratamento cirúrgico em 21 pacientes $(7,2 \%)$, sendo 14 deles do grupo com anel maior que $10 \mathrm{~mm}$ (correspondendo a 13\% do grupo) e 07 do Grupo com anel até $10 \mathrm{~mm}$ (3,8\% do total do grupo).

\section{DISCUSSÃO}

Existem dúvidas por parte de alguns autores ${ }^{15,16}$ se realmente há necessidade de utilização de material protético para restringir o esvaziamento gástrico. Fobi ${ }^{17}$ e Capella ${ }^{11}$ advogam o uso de material para restrição, o primeiro utiliza anel de silicone e o segundo fita de polipropileno e são enfáticos em dizer que caso não se empreguem tais artifícios os pacientes poderão não perder o peso esperado, ou poderão ainda, recuperar parte do peso perdido nos anos seguintes à operação.

Existem autores que não empregam método de restrição. Preferem realização de anastomose calibrada, seja manual ou realizada através de grampeador cirúrgico, como Stahl ${ }^{18}$ que comparou anastomose com grampeador de dois diâmetros de 21 e $25 \mathrm{~mm}$ e não encontrou diferença significativa no emagrecimento.

A derivação gástrica em Y-de-Roux foi a técnica escolhida por nós para a realização do presente estudo, é a operação que realizamos com maior freqüência em nosso Serviço. Fizemos análise apenas das operações feitas por acesso laparotômico porque as que realizamos pela via laparoscópica com cinco anos de seguimento são em número pequeno.

Foram operados 327 pacientes, porém o acompanhamento completo em cinco anos de pós-operatório foi conseguido em 291 pacientes, dos quais foram coletados os dados. Nosso seguimento foi de $89 \%$. Consideramos bom, já que outros estudos relatam taxas inferiores ${ }^{19,20}$ talvez seja porque nosso serviço tenha sido o pioneiro em nossa região e isto fez com que os pacientes fossem mais assíduos nos retornos.

O uso de material de restrição ao esvaziamento gástrico começou com Linner ${ }^{21}$ que reforçava a gastroenteroanastomose com anel de silicone, na época empregado com o intuito de evitar dilatação da bolsa gástrica. Diversos materiais foram empregados com a mesma finalidade, até que Fobi ${ }^{10}$ passou a usar o material protético em posição mais proximal na bolsa gástrica e observou que além de evitar a dilatação do estômago, o anel colocado nessa posição proporcionava maior perda de peso. Tal afirmação foi corroborada por outro estudo ${ }^{11}$.

Enquanto há consenso entre os cirurgiões que a derivação gástrica é excelente para o tratamento da obesida$\mathrm{de}^{14,22}$, há também concordância em relação ao tamanho da bolsa gástrica ${ }^{23,24} \mathrm{o}$ mesmo não pode ser dito sobre o tamanho da anastomose gastrojejunal ${ }^{14,22}$. Alguns cirurgiões pensam que anastomose pequena é fator crucial para o sucesso da operação ${ }^{15}$, outros acreditam que não ${ }^{16}$. Stahl ${ }^{18}$ comparou o diâmetro de anastomose de $21 \mathrm{~mm}$ com $25 \mathrm{~mm}$, realizada mecanicamente sem emprego de anel de constrição em 31 pacientes e não verificou diferença significativa na perda de peso, ressaltando que o tamanho da anastomose é apenas questão de opção do cirurgião.
Determinados autores ${ }^{13,17}$ são enfáticos em afirmar a necessidade de colocação de anel constritor, pois a anastomose, seja ela confeccionada manual ou mecanicamente irá dilatar-se com o tempo, isto talvez explique os resultados do estudo relatado anteriormente.

A colocação das bandas protéticas na forma de anel, situadas a montante da anastomose gastro-jejunal é imputada como fator determinante de sucesso na perda de peso $^{25,26}$, embora nem todos concordem com esta afirmação ${ }^{18}$. Faltam trabalhos avaliando as derivações com ou sem anel e se o fator restritivo imposto por essa bandagem realmente interfere na perda final do peso. Com o objetivo de estabelecer a real importância do uso da bandagem com anel realizamos a presente pesquisa. Empregamos no início anel de 6,2cm de extensão, porém para tentar evitar dificuldades alimentares tivemos a idéia de aumentar seu comprimento, isto determinaria aumento do seu diâmetro e melhor adaptação alimentar. Poderia haver prejuízo na perda do excesso de peso. Isto ficou demonstrado no gráfico, onde observamos que há menor perda de peso no grupo com anel de diâmetro maior.

Quando tivemos a idéia de realizar esta pesquisa, deparamos com o problema de como medir corretamente o diâmetro interno do anel. O anel é colocado ao redor do estômago, envolvendo a parede gástrica que tem espessura variável de pessoa para pessoa, além do que um pouco de gordura pode se interpor entre o estômago e o anel. Tivemos o cuidado de evitar isso. $\mathrm{O}$ anel é calibrado sobre sonda de $10 \mathrm{~mm}$ de diâmetro, isto deveria determinar diâmetro interno fixo, mas não é o que vimos. Assim, usamos o método endoscópico para medi-lo, porém aqui mais um problema surgiu, pois não existe maneira objetiva de fazer esta medida, ela sempre é feita de maneira empírica. $\mathrm{O}$ fato de ser feita por endoscopista experiente e conhecedor da técnica cirúrgica é fundamental. Reconhecemos ser um ponto discutível no estudo, porém como todos os exames foram realizados pelo mesmo examinador acreditamos que esse viés possa não existir. Diversos estudio$\operatorname{sos}^{18,27}$ comparam o resultado de bandagens com tamanhos previamente conhecidos, porém não concordamos com isso, uma vez que a espessura da parede gástrica varia e pode haver interposição de gordura ou vasos entre o anel e a parede do estômago, dessa forma preferimos medir o diâmetro interno do anel por julgarmos ser mais fidedigno. Aqui reside a importância deste estudo, que é inédito neste tipo de medida do anel.

Crampton ${ }^{28}$ comparou dois grupos de pacientes em que empregou anel de $4,5 \mathrm{~cm}$ de extensão com outro em que o comprimento do anel foi de $6,0 \mathrm{~cm}$ e verificou maior perda de peso em dois anos no grupo com anel mais curto. Este estudo não mediu o diâmetro interno do anel, embora possamos inferir que aquele grupo com anel mais longo tivesse diâmetro interno maior. Quando o mesmo autor comparou anéis de 5,5 e $6,0 \mathrm{~cm}$ não encontrou diferença significativa na perda de peso em longo prazo, ressaltamos que mais uma vez o diâmetro interno não fora mensurado, talvez pela dificuldade em fazêlo. Apesar da subjetividade na mensuração acreditamos que esta é a maneira mais exata, pois após a colocação do anel, seu diâmetro interno pode variar em decorrência dos fatores já relatados. 
Fobi ${ }^{29}$, em 2005, comparou o uso de anel de silicone de 4,5 cm de extensão com $6 \mathrm{~cm}$, e constatou que os anéis mais longos proporcionaram maior tolerância à ingestão de alimentos sólidos, conforme verificado no nosso estudo.

O aumento do diâmetro do anel prejudica a perda do excesso de peso de maneira significativa. Deixamos de colocar anel mais frouxo devido estes resultados. Tivemos falha do tratamento cirúrgico $(7,2 \%)$ maior que a relatada na literatu$\mathrm{ra}^{26}$ (em torno de $5 \%$ ), e esta falha foi maior para o grupo com anel de diâmetro maior que $10 \mathrm{~mm}$ ( $13 \%$ contra $3,8 \%$ no grupo com anel menor que $10 \mathrm{~mm}$ ).

A ausência do anel de restrição pode não resultar em emagrecimento maior no início, porém após três anos pode haver maior aumento de peso para os pacientes que não tem essa prótese conforme demonstra o estudo de MacLean ${ }^{12}$, que obser- vou aumento de peso nos pacientes após cinco anos da operação. Outros autores ${ }^{10,30}$ observaram ganho de peso em até $20 \%$ dos pacientes após três anos da operação, sem colocação de prótese constritora, por isso escolhemos análise de cinco anos. Nossos pacientes tiveram pequeno ganho de peso após dois anos do procedimento cirúrgico, porém sem significância estatística comparando-se os resultados de cinco anos com os de um ano de pós-operatório, para ambos os Grupos (Figura 1).

A amostra dos dois Grupos é muito semelhante em relação ao sexo, idade e IMC, isso nos leva a dizer que nossos resultados não foram influenciados por diferença de amostragem.

As condições de realização do presente estudo permitem concluir que: - o diâmetro do anel tem influência na porcentagem de perda do excesso de peso.

\begin{abstract}
Background: The aim of the study is to state if the ring used in the Roux-en-Y gastric bypass is related to the weight loss after surgery. Methods: The study has a 5 years follow up. After endoscopic examination the patientes were divided in two groups: internal diameter of the ring till 10mm (184 patients) and other group with internal diameter of the ring more than $10 \mathrm{~mm}$ (107 patients).The excess of weight loss were measured one, two and five years after surgery and compared one with each other. Results: The excess of weight loss was higher for those patients with tighter ring. Statistc analysis was significant comparing the two groups one, two and five years after surgery, the excess of weight loss was higher in the tighter ring groups for each time after surgery. There were no difference related to sex, age and BMI. Conclusion: The restriction determined by ring increases the excess of weight loss in patients submitted to Roux-en-Y gastric bypass.
\end{abstract}

Key words: Gastroplasty; Obesity morbid; Bariatric surgery.

\section{REFERÊNCIAS}

1. Garner DM, Wooley SC. Confronting the failure of behavioral and dietary treatment of obesity. Clin Psychol Rev. 1991;11(6): 729-41.

2. Patterson EJ, Urbach DR, Swanström LL. A comparison of diet and exercise therapy versus laparoscopic Roux-en-Y gastric bypass surgery for morbid obesity: a decision analysis model. $\mathrm{J}$ Am Coll Surg. 2003;196(3):379-84.

3. Fobi MA, Lee H, Felahy B, Che K, Ako P, Fobi N. Choosing an operation for weight control, and the transected banded gastric bypass. Obes Surg. 2005;15(1): 114-21.

4. Mason EE, Ito C. Gastric bypass in obesity. Surg Clin North Am. 1967;47(6): 1345-9.

5. Mason EE, Printen KJ, Hartford CE, Boyd WC. Optimizing results of gastric bypass. Ann Surg. 1975;182(4): 405-14.

6. Griffen WO Jr, Bivins BA, Bell RM, Jackson KA. Gastric bypass for morbid obesity. World J Surg. 1981;5(6):817-21.

7. Torres JC, Oca CF, Garrison RN. Gastric bypass: Roux-en-Y gastrojejunostomy from the lesser curvature. South Med J. 1983;76910):1217-21.

8. Laws HL, Piantadosi S. Superior gastric reduction procedure for morbid obesity : a prospective, randomized trial. Ann Surg. 1981;193(3):334-40.

9. Linner JR, Drew RL. Technique of anterior wall Roux-en-Y gastric bypass for the treatment of morbid obesity. Contemp Surg. 1985;26:46-59.

10. Fobi MAL, Lee H, Flemming AW. The surgical technique of the banded gastric bypass. J Obes Weight Regul. 1989;8(2):99-102.

11. Capella RF, Capella JF, Mandec H, Nath P. Vertical banded gastroplasty-gastric bypass: preliminary report. Obes Surg. 1999;1(4):389-95.
12. MacLean LD, Rhode BM, Sampalis J, Forse RA. Results of the surgical treatment of obesity. Am J Surg. 1993;165(1):155-62.

13. Capella JF, Capella RF. The weight reduction operation of choice: vertical banded gastroplasty or gastric bypass? Am J Surg. 1996;171(1):74-9.

14. Fobi M. Why the operation I prefer is silastic ring vertical gastric bypass. Obes Surg. 1991;1(4):423-6.

15. Benotti PN, Forse RA. The role of gastric surgery in the multidisciplinary management of severe obesity. Am J Surg. 1995;169(3):361-7.

16. MacLean LD, Rhode BM, Forse RA. Late results of vertical banded gastroplasty for morbid and super obesity. Surgery. 1990;107(1):20-7.

17. Fobi MA, Lee H. Silastic ring vertical banded gastric bypass for the treatment of obesity two years of follow-up in 84 patients [corrected]. J Natl Med Assoc. 1994; 86(2):125-8. Erratum in: J Natl Med Assoc 1994 Jun;86(6):432.

18. Stahl RD, Sherer RA, Seevers CE, Johnston D. Comparison of 21 vs. $25 \mathrm{~mm}$ gastrojejunostomy in the gastric bypass procedureearly results. Obes Surg. 2000; 10(6):540-2.

19. Mason EE. Gastroplasty. Major Probl Clin Surg. 1981;26:386-9.

20. Mason EE. Vertical banded gastroplasty for obesity. Arch Surg. 1982;117(5): 701-6.

21. Linner JH. Gastric operation. In: Linner JH, editor. Surgery for morbid obesity. New York: Springer; 1984. p. 65-107.

22. Crampton NA, Izvornikov V, Stubbs RS. Silastic ring gastric bypass: results in 64 patients. Obes Surg. 1997;7(6):489-94.

23. Howard L, Malone M, Michalek A, Carter J, Alger S, Van Woert J. Gastric bypass and vertical banded gastroplasty- a prospective randomized comparison and 5-year follow-up. Obes Surg. 1995;5(1):114-21. 
24. Fobi M, Lee H, Igwe D, Felahy B, James E, Stanczyk M, Fobi N. Band erosion: incidence, etiology, management and outcome after banded vertical gastric bypass. Obes Surg. 2001;11(6):699-707.

25. Garrido Júnior AB. Variantes técnicas e resultados do tratamento cirúrgico da obesidade mórbida. Arq Bras Cir Dig. 1993;892):17881.

26. Fobi MA. Vertical banded gastroplasty vs gastric bypass: 10 years follow-up. Obes Surg. 1993;3(2):161-4.

27. McDonald KG, Poires WJ. Roux gastric bypass or vertical banded gastroplasty. Probl Gen Surg. 1992;9:321-31.

28. Crampton NA, Izvornikov V, Stubbs RS. Silastic ring gastric bypass: a comparision of two ring sizes: a preliminary report. Obes Surg. 1997;7(6): 495-9.

29. Fobi MA. Placement of the GaBP ring system in the banded gastric bypass. Obes Surg. 2005;15(8):1196-201.
30. Halverson JD, Koehler RE. Assessment of patients with failed gastric operations for morbid obesity. Am J Surg. 1983;145(3):357-63.

Como citar este artigo:

Valezi AC, Brito EM, Souza JC, Guariente AL, Emori FT, Lopes VC. A importância do anel de silicone na derivação gástrica em Y-deRoux para o tratamento da obesidade. Rev Col Bras Cir. [periódico na Internet] 2008; 35(1). Disponível em URL: http://www.scielo.br/ rcbc

Endereço para correspondência:

Antonio Carlos Valezi

Rua Santos 777 apto 1302

86020-041 - Londrina - PR

valezi@sercomtel.com.br 